\title{
Calibration of imaging parameters for space-borne airglow photography using city light positions
}

Yuta Hozumi ${ }^{*}$, Akinori Saito ${ }^{1}$ and Mitsumu K. Ejiri ${ }^{2,3}$

\begin{abstract}
:
A new method for calibrating imaging parameters of photographs taken from the International Space Station (ISS) is presented in this report. Airglow in the mesosphere and the F-region ionosphere was captured on the limb of the Earth with a digital single-lens reflex camera from the ISS by astronauts. To utilize the photographs as scientific data, imaging parameters, such as the angle of view, exact position, and orientation of the camera, should be determined because they are not measured at the time of imaging. A new calibration method using city light positions shown in the photographs was developed to determine these imaging parameters with high accuracy suitable for airglow study. Applying the pinhole camera model, the apparent city light positions on the photograph are matched with the actual city light locations on Earth, which are derived from the global nighttime stable light map data obtained by the Defense Meteorological Satellite Program satellite. The correct imaging parameters are determined in an iterative process by matching the apparent positions on the image with the actual city light locations. We applied this calibration method to photographs taken on August 26, 2014, and confirmed that the result is correct. The precision of the calibration was evaluated by comparing the results from six different photographs with the same imaging parameters. The precisions in determining the camera position and orientation are estimated to be $\pm 2.2 \mathrm{~km}$ and $\pm 0.08^{\circ}$, respectively. The $0.08^{\circ}$ difference in the orientation yields a $2.9-\mathrm{km}$ difference at a tangential point of $90 \mathrm{~km}$ in altitude. The airglow structures in the photographs were mapped to geographical points using the calibrated imaging parameters and compared with a simultaneous observation by the Visible and near-Infrared Spectral Imager of the lonosphere, Mesosphere, Upper Atmosphere, and Plasmasphere mapping mission installed on the ISS. The comparison shows good agreements and supports the validity of the calibration. This calibration technique makes it possible to utilize photographs taken on low-Earth-orbit satellites in the nighttime as a reference for the airglow and aurora structures.
\end{abstract}

Keywords: Camera calibration, Airglow, Space-borne imaging, International Space Station

\section{Introduction}

The International Space Station (ISS) is a unique facility, providing opportunities to conduct various observations of the Earth's upper atmosphere. With the recent development of digital photography technology, a number of photographs of the upper atmosphere that contain the airglow and aurora have been taken by astronauts on the ISS with digital single-lens reflex (DSLR) cameras. These photographs are open to the public and available

\footnotetext{
*Correspondence: hozumi@kugi.kyoto-u.ac.jp

1 Graduate School of Science, Kyoto University, Kyoto, Japan

Full list of author information is available at the end of the article
}

on NASA's Web site (“The Gateway to Astronaut Photography of Earth 1995"). Some of the photographs captured the airglow in the mesosphere and the $F$-region ionosphere on the night side of the Earth. This spaceborne imaging covers much wider geographical area than ground-based imaging observations. Inspired by these photographs, we carried out the Astronaut-Ionosphere, Mesosphere, upper Atmosphere, and Plasmasphere mapping (A-IMAP) campaign observation to investigate the airglow structure of the Earth's upper atmosphere. In this observation, an astronaut took sequential photographs of the airglow through the window of the cupola, an ISS module, while the ISS flew on the night side of the Earth. 
The A-IMAP campaign is associated with the Ionosphere, Mesosphere, upper Atmosphere, and Plasmasphere mapping (IMAP) mission. Two imagers of the IMAP mission, one is for the airglow in visible and infrared light and the other is for resonant scattering from ions in extreme ultraviolet light, are operated almost continuously from September 2012 to August 2015. During the operation period of the IMAP mission, ten experiments of the A-IMAP campaign were carried out in 2014 and 2015.

To utilize the photographs as scientific data, the imaging parameters must be determined. The camera was fixed to the ISS during the experiments, but the orientation of the camera was different for each experiment because the camera was detached from the window of the cupola after the experiment. A zoom lens was used for the A-IMAP campaign, and its focus length and viewing direction were adjusted in each experiment to capture clear airglow structures while minimizing the obstruction by other ISS structures such as a window-frame, robot arms and solar panels. Therefore, the camera orientation and angle of view (AOV) are unknown parameters and must be determined to utilize the image for airglow study. The precise camera position along the ISS trajectory is also an unknown parameter because the clock of the camera is not precise. To determine these imaging parameters, we developed a new calibration technique using the city light positions shown in the photographs. Because observations were conducted at night, city light positions are better reference than topographic features. The location of the camera is an unknown parameter. Therefore, the positions of city lights were used in our method, despite that the positions of stars are generally used to determine the field of view (FOV) of images taken in the nighttime from spacecrafts, rockets, and ground-based instruments. The attitude of a spacecraft can be determined by the position of stars; however, the positions of the spacecraft cannot be determined by stars because the distant star does not have a detectable parallax. The distances between the spacecraft and cities are close; hence, the city light position in the image gives rise to a detectable parallax, which is necessary for calibration of the camera location.

This report is organized as follows. The method of calibration is described in the next section. In Results and discussion section, we present the result of applying the method to photographs taken on August 26, 2014, and evaluate precision of the calibration. Then, we map the airglow structures in the photographs to the geographical coordinates using the calibrated imaging parameters and discuss their vertical and horizontal structures. A comparison with a simultaneous observation of the 630$\mathrm{nm}$ airglow emission by another instrument of the ISS/ IMAP mission will also be presented. The conclusion of this report is presented in the last section.

\section{Methods}

We developed a new method for calibrating imaging parameters using the city light positions shown in the airglow photographs taken from a low-Earth-orbit (LEO) satellite. The apparent city light positions were compared with the actual city light positions on the Earth by applying the pinhole camera model. The actual positions were determined by the global nighttime stable light data obtained by the visible and infrared sensors (OLS) on the Defense Meteorological Satellite Program (DMSP) satellite (Version 4 DMSP-OLS Nighttime Lights Time Series 2015). The resolution of the light data is $1 \mathrm{~km}$. In the pinhole camera model (e.g., Trucco and Verri 1998; Jaehne 1997), the relation between the city light position on Earth and that on the photograph is given by

$$
\begin{aligned}
& {\left[\begin{array}{l}
x \\
y \\
z
\end{array}\right]=R\left[\begin{array}{l}
X \\
Y \\
Z
\end{array}\right]-t,} \\
& u=f \frac{x}{z}+C_{x}, \\
& v=-f \frac{y}{z}+C_{y},
\end{aligned}
$$

where $\left[\begin{array}{lll}x & y & z\end{array}\right]^{T}$ is the coordinates of the city light in the camera coordinate system, which is the coordinate system fixed to the camera, and $\left[\begin{array}{lll}X & Y & Z\end{array}\right]^{T}$ is the coordinates of the city light in the world coordinate system. In Eq. (1), $t$ is the camera position in the world coordinates and $R$ is the rotation matrix corresponding to the orientation of the digital camera. The matrix $R$ transforms the coordinates of a point to the coordinate system fixed to the camera. In Eqs. (2) and (3), $(u, v)$ is the coordinates of the projection point in the photograph in units of pixel and $f$ is the focal length expressed in units of pixel, which varies as a function of AOV as $f=\frac{1}{\tan \left(\frac{\mathrm{AOV}}{2}\right)} \frac{N_{\mathrm{pix}}}{2}$, where $N_{\text {pix }}$ is the number of horizontal pixels. Moreover, $\left(C_{x}, C_{y}\right)$ is the coordinates of the center of the photograph in units of pixel. The Euler angles $\theta, \sigma$, and $\varphi$ are used to describe the orientation of the camera relative to the ISS. The relation between $R$ and the ISS orientation $R_{\text {ISS }}$ can be written in terms of the Euler angles as

$$
\begin{aligned}
R= & {\left[\begin{array}{ccc}
\cos \varphi & \sin \varphi & 0 \\
-\sin \varphi & \cos \varphi & 0 \\
0 & 0 & 1
\end{array}\right]\left[\begin{array}{ccc}
\cos \sigma & 0 & -\sin \sigma \\
0 & 1 & 0 \\
\sin \sigma & 0 & \cos \sigma
\end{array}\right] } \\
& {\left[\begin{array}{ccc}
1 & 0 & 0 \\
0 & \cos \theta & \sin \theta \\
0 & -\sin \theta & \cos \theta
\end{array}\right] R_{\mathrm{ISS} .} }
\end{aligned}
$$


Following the formulation of Brown (1971), we assume the radial distortion of the lens is given by

$$
\begin{aligned}
& u_{\text {corrected }}=u\left(1+k_{1} r^{2}+k_{2} r^{4}\right), \\
& v_{\text {corrected }}=v\left(1+k_{1} r^{2}+k_{2} r^{4}\right),
\end{aligned}
$$

where $r$ is the distance from the center of the image in units of pixel, and $k_{1}$ and $k_{2}$ are coefficients of the radial distortion. With these equations, we can convert the city light positions in the world coordinates to projection points on the image.

The camera location was assumed to be the same as the ISS location. Although the location of the ISS is known as a function of time, the times at which the photographs are taken are not precise. The time when the photograph was captured was recorded in the metadata of the image file in the exchangeable image file format. However, the clock of the camera is not accurate and has a time lag with respect to Universal Time (UT) because the clock was set by hand. It is necessary to determine the time lag so as to obtain the exact location of the camera. Because the orientation of the ISS is also known as a function of time, the orientation of the camera can be expressed in terms of its orientation relative to the ISS. Therefore, the unknown parameters are the time lag, orientation of the camera relative to the ISS, AOV, and the coefficients of lens distortion $k_{1}$ and $k_{2}$.

The correct set of these imaging parameters was determined by applying the imaging model described above. Once the set of parameters is assumed, the actual city light location can be converted to a position on the photograph using Eqs. (1-6). To evaluate the correctness of the assumed parameters, the mean distance between the city light positions on the photograph and projected positions were calculated. This process was iterated over the parameter space to determine the best set of parameters that minimizes the mean distance.

The total number of unknown parameters is seven, i.e., one for the time lag, three for the orientation, one for the AOV, and two for the lens distortion. Each city light position on the two-dimensional plane of a photograph provides two restrict conditions. Therefore, at least four city light positions are necessary for the calibration. Practically, more than four city light positions are used to determine the parameters with improved accuracy.

\section{Results and discussion}

\section{Calibration results}

We applied the calibration method to the photographs from the A-IMAP mission taken on August 26, 2014. On the day, the ISS flew on the night side of the Earth at latitude $21.8^{\circ} \mathrm{N}$ and longitude $93.2^{\circ} \mathrm{E}$ over Myanmar at 13:38 UT and flew out of the night side at latitude $49.2^{\circ} \mathrm{S}$ and longitude $142.5^{\circ} \mathrm{W}$ over the east of New Zealand at 14:12 UT. During the night path of the ISS, photographs were taken with a DSLR camera (Nikon D3s) and a lens (AI AF-s Zoom-Nikkor 17-35 mm f/2.8D IF-ED). Four photographs were taken every $11 \mathrm{~s}$ with an exposure time of $2.5 \mathrm{~s}$. The camera was operated in Manual mode with an f-number of 2.8 and ISO speed of 102,400. Each photograph has $4256 \times 2382$ pixels. An image of the photographs is shown in Fig. 1. The observation time recorded in the metadata is 13:47:00.08 UT. This photograph is hereafter referred to as photograph A. The Earth's limb was captured in the upper part of the image. Above the Earth's limb, two airglow layers are identified. The lower layer is caused by emission from the mesopause region, and the upper layer is caused by emission from the $F$-region ionosphere. On the Earth's surface, several city lights are seen in the image. City lights of the islands of Indonesia in the center of the image and those of the northwest coast of Australia on the right side of the image are recognized. The green circles indicate city light positions used for the calibration. The city lights in the middle and long distance ranges from the ISS were chosen. The city lights too close to the ISS had a large apparent size on the photograph, leading to a reduction in positioning accuracy. The positions of the selected city lights should be widely distributed in the image to improve accuracy of the calibration. Because of the ISS's motion during the exposure time, $2.5 \mathrm{~s}$, the apparent images of the city lights are stretched and have line-shape along the ISS trajectory as seen in Fig. 1. This effect is appar-

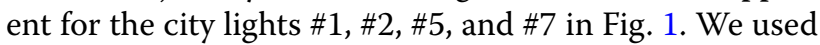
the low/near end of the line-shaped image of these city

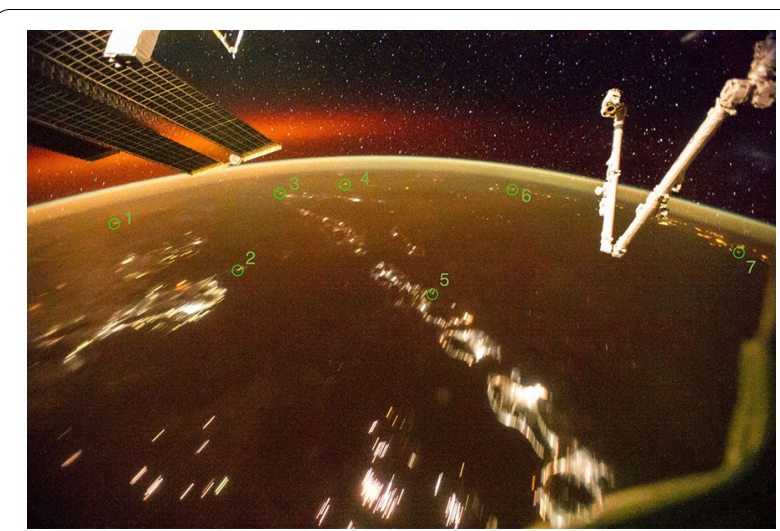

Fig. 1 One of the photographs taken on August 26, 2014 (Photograph A). The recorded time is 13:47:00.08 UT. The green circles indicate city light positions used for the calibration. The green numbers are corresponding to the red numbers shown in Fig. 2 
lights, which correspond to the positions of city lights at the time of end of exposure time. Figure 2 shows the nighttime stable lights map derived from the DMSP-OLS data for the year 2013 (2) with the city locations indicated by the red circles. We utilized the data for the year 2013 to identify the city lights positions, ignoring the variation between stable lights for the year 2013 and city lights on the day August 26, 2014. City light covered by clouds is obscurely imaged from the ISS and should not be used for the calibration. The coverage of clouds is easily identified by comparison between A-IMAP photographs and DMSP-OLS data.

The calibration method was applied to photograph A. The mean distance between the city light position on the photograph and projected positions was calculated for every node of seven-dimensional parameter space with resolutions of $0.1 \mathrm{~s}$ for the time lag, $0.01^{\circ}$ for the Euler angles and AOV, $10^{-10}$ for $k_{1}$, and $10^{-17}$ for $k_{2}$. The parameter set minimizing the mean distance is the best set. The derived parameters are listed in the first row of Table 1 . Figure 3 shows the parameter dependence of the mean distance. The variation of the mean distance as a function of each assumed parameter is calculated, while the other parameters are kept to be the best. Because each parameter has a smooth curve and a sharp minimum, as shown in Fig. 3, the searching resolution of each parameter for the iteration process is valid.

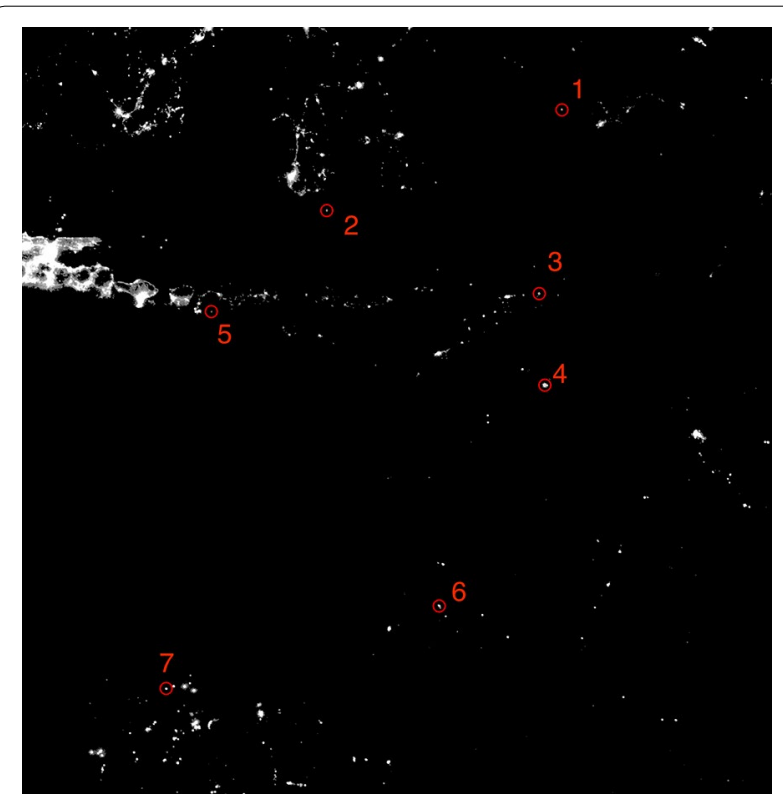

Fig. 2 City lights map observed by the DMSP satellite. The red circles indicate city light positions used for the calibration. The red numbers are corresponding to the green numbers shown in Fig. 1. The DMSP image and data were processed by NOAA's National Geophysical Data Center, and the DMSP data were collected by US Air Force Weather Agency
To evaluate the precision and stability of the calibration method, we applied the method to other five photographs for different city light conditions. These five photographs were taken in the same night path of the ISS with 2-min time interval from 13:49 to 13:57 UT using the same camera and the same setting as for photograph A. Photographing time recorded in the metadata for each photograph is presented in Table 1 (photographs B-F). During this period, the focal length of the lens was not changed and the camera orientation was stable relative to the ISS, because the camera was fixed to the ISS. The time lag of the camera's clock could be regarded as stable with 0.1 -s resolution in this 10 -min period. Therefore, the imaging parameters derived from these five photographs should be the same as those derived from photograph $\mathrm{A}$. The distance between adjacent photographing positions for these six photographs was about $880 \mathrm{~km}$, and the cities used in each calibration process were not used in the calibrations for the other photographs. The calibration result and their standard deviation are presented in Table 1 . The time lag was estimated in the range from -15.5 to $-16.3 \mathrm{~s}$ with a standard deviation of $0.3 \mathrm{~s}$. The camera position along the ISS orbit was derived from the time lag. A standard deviation of $0.3 \mathrm{~s}$ was corresponding to $2.2 \mathrm{~km}$ in the horizontal distance along the ISS trajectory, considering the ISS speed of $7.4 \mathrm{~km} / \mathrm{s}$. Therefore, the calibration precision for the camera position was $\pm 2.2 \mathrm{~km}$. The standard deviations of the derived Euler angles $\theta, \sigma$, and $\varphi$ for the orientation of the camera were determined with a standard deviations within $0.08^{\circ}$, yielding a $2.9-\mathrm{km}$ difference in the plane perpendicular to the line of sight (LOS) at the tangential point of $90 \mathrm{~km}$ in altitude. The $2.9 \mathrm{~km}$ distance was the calibration precision for the determination of the altitude profiles of the airglow on the limb. The AOV was derived in the range from $90.53^{\circ}$ to $91.93^{\circ}$. The distortion coefficients $k_{1}$ and $k_{2}$ were estimated in the ranges from $-4.26 \times 10^{-8}$ to $-0.97 \times 10^{-8}$ and from $-0.26 \times 10^{-15}$ to $10.55 \times 10^{-15}$, respectively. The AOV, $k_{1}$, and $k_{2}$ are not independent parameters. They are related to each other, and they determine the FOV and its distortion. To evaluate their combined expression, we calculated the angle between the LOS of the pixel at the center of image and that of the pixel with a distance of 1500 pixel from the center. We denote this angle by $\alpha$, and the derived values of $\alpha$ are listed on the rightmost column of Table 1. The derived values of $\alpha$ were in the range from $36.71^{\circ}$ to $36.99^{\circ}$ with a standard deviation of $0.12^{\circ}$. The calibration precision for the FOV was $\pm 0.12^{\circ}$. The calibration errors would be mainly attributed to the errors in determination of city light position on the photograph. Because each city light has a finite size on the photographs, the determination of its position yields some errors. 
Table 1 Imaging parameters determined by the calibration

\begin{tabular}{|c|c|c|c|c|c|c|c|c|c|c|}
\hline & $\begin{array}{l}\text { Recorded } \\
\text { time (UT) }\end{array}$ & $\begin{array}{l}\text { \# of city } \\
\text { lights }\end{array}$ & Time lag (s) & $\theta$ (deg.) & $\psi$ (deg.) & $\varphi$ (deg.) & AOV (deg.) & $k_{1}\left(\times 10^{-8}\right)$ & $k_{2}\left(\times 10^{-15}\right)$ & $a$ (deg. \\
\hline Photo. A & 13:47:00.08 & 7 & -16.3 & 34.93 & -9.56 & 9.52 & 91.83 & -0.97 & -0.26 & 36.72 \\
\hline Photo. B & 13:49:01.16 & 7 & -15.7 & 34.97 & -9.64 & 9.50 & 91.60 & -1.79 & 1.35 & 36.92 \\
\hline Photo. C & 13:51:02.16 & 7 & -16.3 & 34.93 & -9.62 & 9.46 & 91.93 & -1.20 & 0.98 & 36.73 \\
\hline Photo. D & 13:53:00.41 & 8 & -15.9 & 34.96 & -9.76 & 9.38 & 91.37 & -2.57 & 5.20 & 36.71 \\
\hline Photo. E & 13:55:01.32 & 7 & -15.5 & 35.10 & -9.72 & 9.54 & 91.25 & -2.42 & 4.29 & 36.70 \\
\hline Photo. F & 13:57:02.49 & 7 & -16.2 & 34.84 & -9.65 & 9.53 & 90.53 & -4.16 & 10.55 & 36.99 \\
\hline $\begin{array}{l}\text { Standard devia- } \\
\text { tion }\end{array}$ & & & 0.3 & 0.08 & 0.07 & 0.05 & 0.24 & 1.06 & 4.63 & 0.12 \\
\hline
\end{tabular}

The standard deviations of these derived parameters for photograph A-F are listed in the last row

Stars are also captured in the image. The orientation of the camera can be determined from star positions. For the current case, however, stars are captured only in small upper portion of the image and city lights are captured more widely in the image. The calibration precision evaluated above seems sufficient for most studies of airglow. Therefore, we use only city light positions for the calibration of A-IMAP observations. However, additional usage of star map for determination of camera orientation will provide better calibration accuracy for future other observations when stars are distributed widely in the image.

The calibration results are stable in the ISS path, along which the imaging parameters are not changed. Therefore, only one photograph is needed when applying the method to determine the imaging parameters for sequential photographs. Even though most photographs taken along the ISS path did not have clear city lights, because of clouds or the land-sea distribution under the ISS path, calibration of the imaging parameters is possible if there were more than one photograph with an enough number of city lights.

\section{Vertical airglow structures}

The airglow structures on the photographs were mapped to geographical locations with the derived imaging parameters and compared with previous studies so as to evaluate the validity of the calibration results.

The camera had a CMOS image sensor that contains a red, green, and blue color filter of the Bayer arrangement (Bayer 1976). The number of pixels for the green channel was 2142 in width and 2844 in height, and that for the red and blue channels was 2142 in width and 1422 in height. A laboratory experiment was conducted to obtain the sensitivity spectrum for each color filter. Thirty monochromatic lines from 400 to $700 \mathrm{~nm}$ with a bandpass of $2 \mathrm{~nm}$ were used to measure the spectral response of the sensor. The camera was operated in manual mode with 2.5 -s exposure time and 102,400 ISO speed. The mean raw count per pixel per $\mathrm{cd} / \mathrm{m}^{2}$ for each monochromatic line is shown in Fig. 4. The counts were normalized by divided by the peak mean count of the green channel. The result showed that the sensor is sensitive to light of wavelengths from 410 to $680 \mathrm{~nm}$.

The spectra of the airglow have been observed from ground (e.g., Krassovsky et al. 1962, Broadfoot and Kendall 1968) and space (Broadfoot and Bellaire 1999). The responsivity of each color channel to the airglow can be estimated by comparing the camera sensitivity spectra with the airglow spectra. The red channel has sensitivity in the range from 570 to $680 \mathrm{~nm}$. In this wavelength range, the intense emissions of the airglow are atomic oxygen emission $(\mathrm{OI})$ at $630 \mathrm{~nm}$, sodium emissions $(\mathrm{NaD})$ in the range of 588.9-589.6 $\mathrm{nm}$, and hydroxyl $(\mathrm{OH})$ emissions. Therefore, the red channel had a capability to measure these three emissions. The green channel had sensitivity in the range from 460 to $620 \mathrm{~nm}$ with a peak near the OI 557.7-nm green line emission. The OI green line dominated the green channel, although $\mathrm{OH}$ and $\mathrm{NaD}$ emissions also contributed to the green channel. In the blue channel sensitivity range from 410 to $540 \mathrm{~nm}$, no intense emission line of the airglow was identified. However, the emissions of the $\mathrm{O}_{2}$ Herzberg I, II, and II and the Chamberlain system (Chamberlain 1958; Khomich et al. 2008) could be measured in the blue channel.

The limb profile of the raw count for photograph A was derived using the imaging parameters determined by the calibration method. The imaging data were averaged over 20 pixels along the horizontal axis of the image so as to increase the signal-to-noise ratio. Although the horizontal axis of the image is not perfectly identical to the Earth's horizontal axis, the two axes are roughly parallel as shown in Fig. 1. Because of the Earth's curvature, there is $0.9-\mathrm{km}$ difference in tangential altitude between the right and left side pixels of the 20 pixels around $90 \mathrm{~km}$. We ignored this discrepancy of the two axes in the horizontal 20 pixels and averaged data along the horizontal axis of the image to reduce the calculation quantity. 

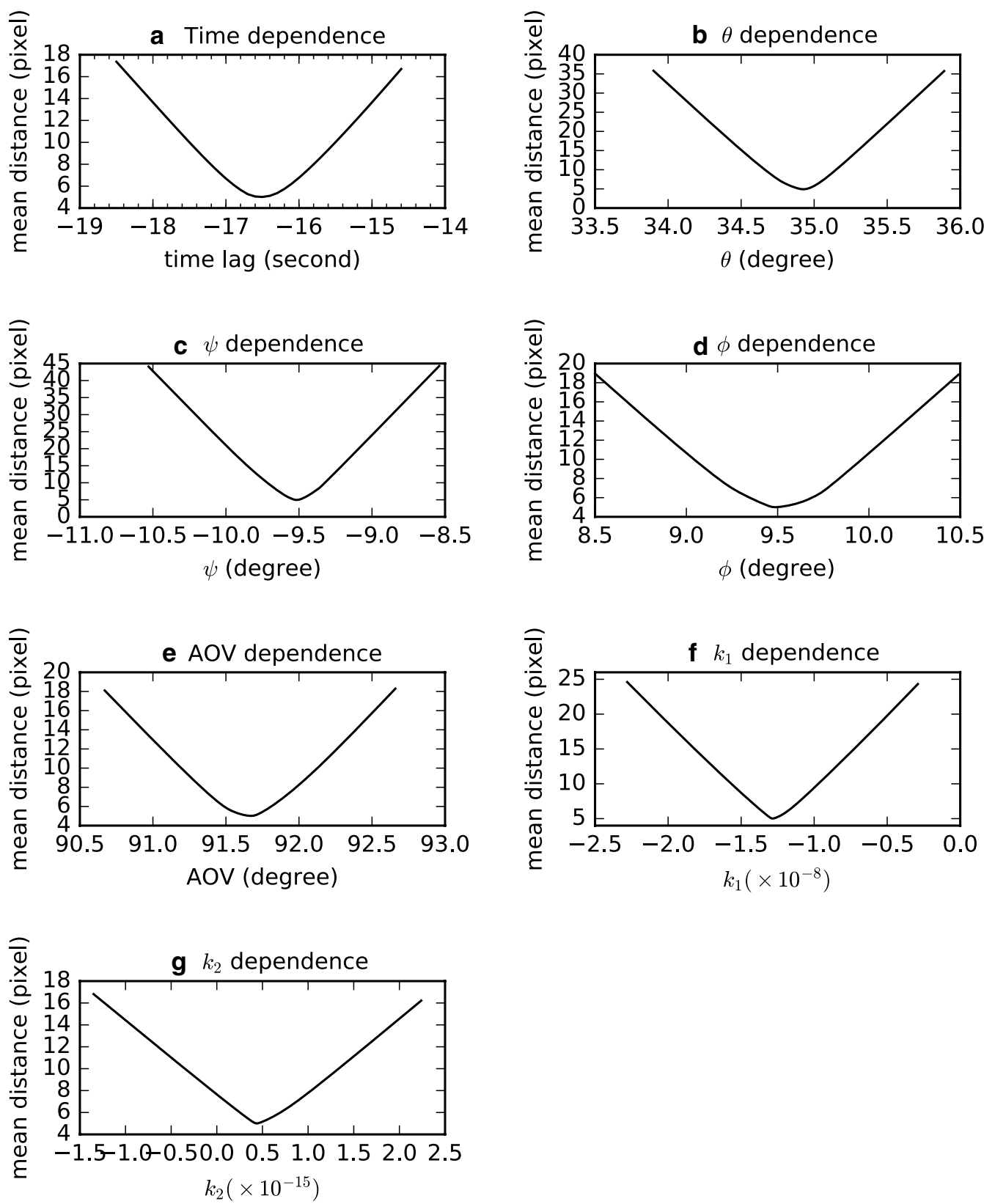

Fig. 3 Parameter dependence of the mean distance. a-g panels show the time lag, $\theta, \psi, \phi, A O V, k_{1}$ and $k_{2}$ dependences, respectively. Each curve has a clear minimum peak

The pixels with counts more than the average count by its standard deviation were removed so as to avoid contamination from stars. We calculated the imaging parameters from those determined using photograph A (Table 1). The limb profile as a function of tangential altitude is plotted in Fig. 5. The error bars indicate the standard deviation of the averaged 20 pixels. The coordinates of the tangential point at $90 \mathrm{~km}$ in altitude were $13.1^{\circ}$ $\mathrm{S}$ and $130.3^{\circ} \mathrm{E}$ in geographical coordinates. The green channel was found to have 102 pixels in the range from 0 to $100 \mathrm{~km}$ in the tangential altitude, and the red and blue channels were found to have 51 pixels in the same range. Therefore, the height resolution for the green channel was $1.0 \mathrm{~km}$ and those for the red and blue channels were $2.0 \mathrm{~km}$. The green and red channels had a broad peak in the range from 180 to $300 \mathrm{~km}$. The 557.7 and $630-\mathrm{nm}$ OI emissions from the $F$-region ionosphere were considered to be the cause for the peaks in the green and red 


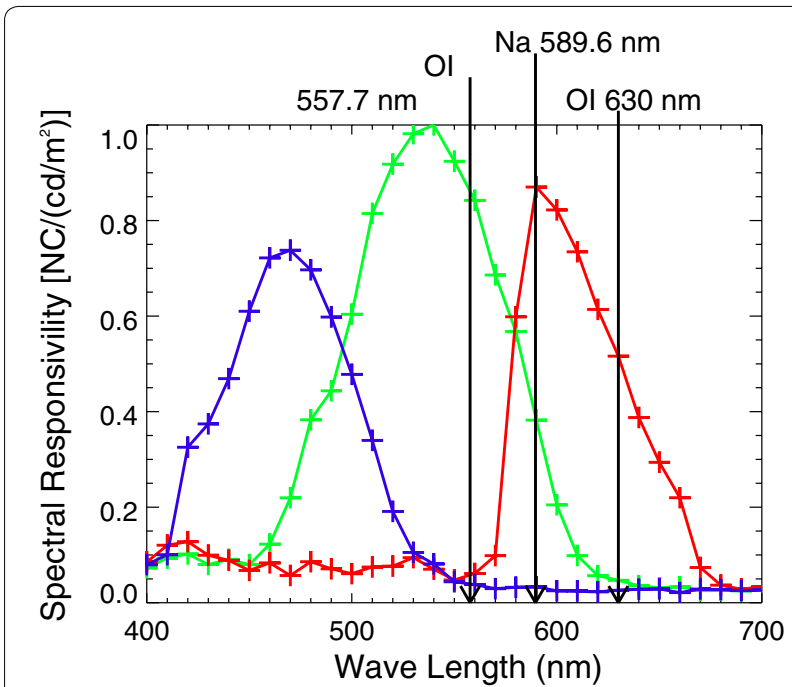

Fig. 4 Sensitivity spectrum of the camera obtained from the laboratory experiment. The response of the sensor to monochromatic light was examined in 10-nm intervals. The mean raw count per pixel per $\mathrm{cd} / \mathrm{m}^{2}$ is normalized by divided by the peak mean count of the green channel. The wavelengths of the major emission lines are also plotted

channels, respectively. A previous study showed that the 630-nm OI airglow emission is in the altitude range of $180-350 \mathrm{~km}$ and the low- and midlatitude regions based on data of rockets (Takahashi et al. 1990) and satellites (Adachi et al. 2010). The altitude of the 630-nm emission derived from ground-based imaging network with the triangulation technique was reported to be $260 \pm 10 \mathrm{~km}$ in middle latitude (Kubota et al. 2000). The 557.7-nm emission in the $F$-region has an intensity that is $30 \%$ of that of the 630-nm emission (Khomich et al. 2008). The vertical profiles of the $F$-region emission obtained from the red and green channels of the A-IMAP observation have a good agreement with these studies. All of the channels had a clear peak around an altitude of $90 \mathrm{~km}$. The red channel had a peak at an altitude of $86 \mathrm{~km}$. The $\mathrm{NaD}$ and $\mathrm{OH}$ emissions in the mesopause region were possible causes for the red channel peak. The green channel had a peak at an altitude of $94 \mathrm{~km}$. It was mainly dominated by the $557.7-\mathrm{nm}$ OI airglow emission in the mesopause region. The $\mathrm{NaD}$ and $\mathrm{OH}$ airglow would also contribute to the green channel profile in this altitude range to some extent. The peak altitude of the emissions observed at the mesopause altitude had a good agreement with previous observations by rockets (e.g., Packer 1961; Baker and Stair 1988) and satellites (e.g., Liu and Shepherd 2006). According to those studies, the typical peak altitudes are $95 \mathrm{~km}$ for the $557.7-\mathrm{nm}$ OI emission, $90 \mathrm{~km}$ for $\mathrm{NaD}$ emissions, and $86 \mathrm{~km}$ for $\mathrm{OH}$ airglow. The emission layer in the blue channel with a peak latitude of $94 \mathrm{~km}$ could be attributed to the $\mathrm{O}_{2}$ emission of Herzberg I, II, and III,

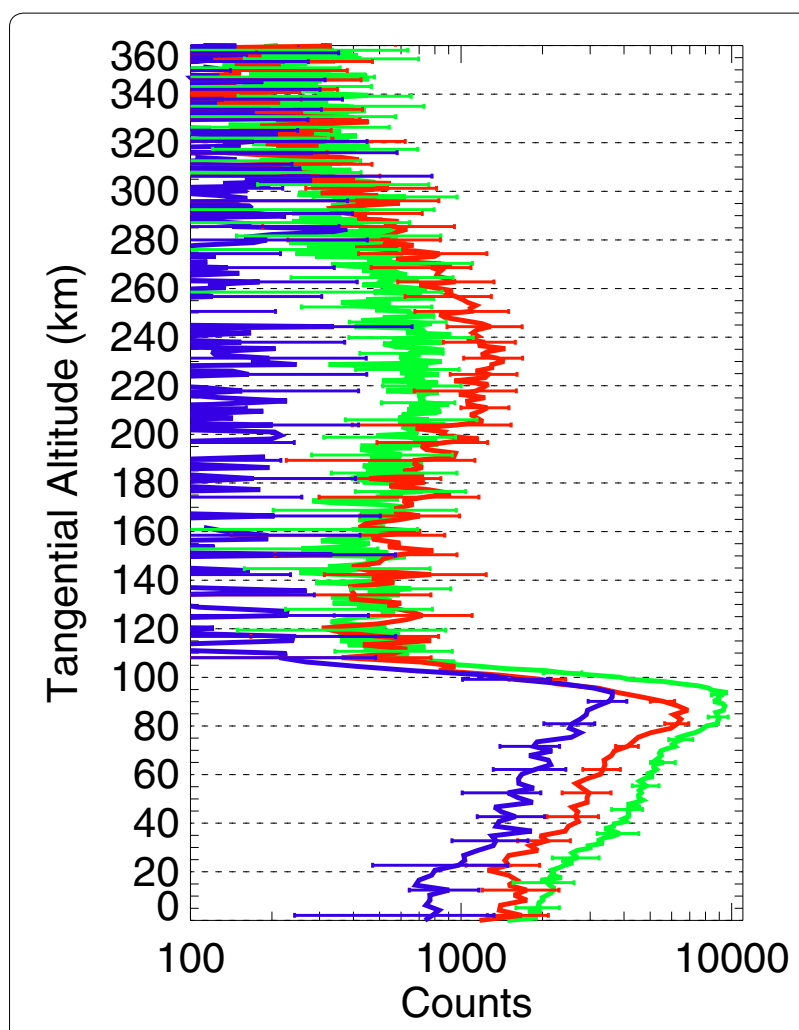

Fig. 5 Limb profiles of the three channels derived from photograph A. The coordinates of the tangential point at $90 \mathrm{~km}$ were $12.6^{\circ} \mathrm{S}$ and $130.5^{\circ} \mathrm{E}$. The recoded time is 13:47:00.08 UT. The raw counts of the pixels along the vertical line of the image are averaged to 10 pixels horizontally so as to increase the signal-to-noise ratio and are plotted as a function of tangential altitude. The limb profile has its tangential point at $32.3^{\circ} \mathrm{S}$ and $124.2^{\circ} \mathrm{E}$ and $90 \mathrm{~km}$ in altitude. The error bars show the standard deviation of the averaged pixels. The green, red, and blue lines indicate the green, red, and blue channels, respectively

and/or the Chamberlain system (Khomich et al. 2008). The peak altitude of Herzberg I and II and the Chamberlain system was reported to be in the altitude range from 94 to $100 \mathrm{~km}$ (López-González et al. 1992). The vertical structures of the airglow captured in the three channels are consistent with previous studies.

\section{Horizontal airglow structures}

We derived the horizontal structure of the Equatorial Ionization Anomaly (EIA) of the 630-nm OI emission from the red channel of the sequential photographs of the A-IMAP campaign and compared it with a simultaneous observation of the 630-nm emission by the Visible and near-Infrared Spectral Imager (VISI), one of the instruments of the ISS-IMAP mission.

In order to increase the signal-to-noise ratio and reduce the calculation quantity, we compressed the 
horizontal axis of the A-IMAP image from 2142 to 214 pixels by averaging data. The raw counts of the red channel of the photographs were averaged over 20 pixels for every 10 pixels along the horizontal axis of the image. Because the discrepancy between the horizontal axis of the image and the Earth's horizontal axis is small in the range of horizontal 20 pixels, we can safely average data of the red channel in 20 pixels along the horizontal axis of the image. In the averaging process, the contamination by stars was removed with the same method as mentioned above. For each vertical profile along the vertical axis of the A-IMAP image, the total row count of the red channel in the range from 180 to $300 \mathrm{~km}$ in tangential altitude was calculated and mapped to its tangential point at $250 \mathrm{~km}$ in altitude. This process was applied to 570 photographs taken from 13:38:59 to 14:05:09 UT on August 26, 2014. The result is presented in Fig. 6a with a black-red color scale. The ISS flew toward southeast and the center LOS of the camera pointed southeast with an $\mathrm{AOV}$ of $92^{\circ}$ in the low-latitude region at that time. The width of the FOV across the track was about $2100 \mathrm{~km}$ at $250 \mathrm{~km}$ in altitude. The horizontal resolution along the

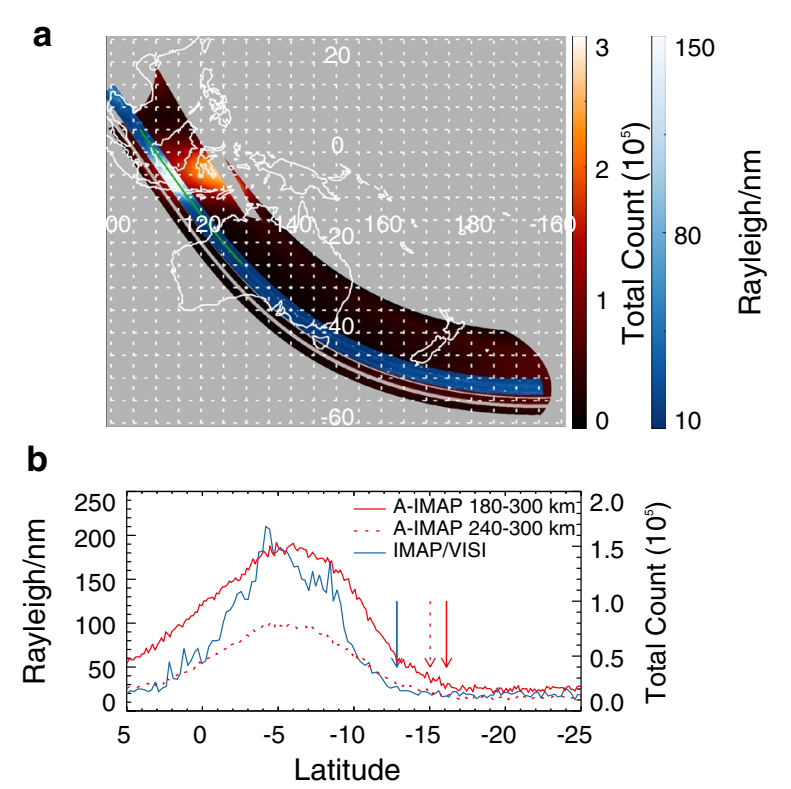

Fig. 6 Mapped total counts and the IMAPNISI data. In a, total counts of the red channel of the photographs in the range between tangential altitudes of 180 and $300 \mathrm{~km}$ are mapped to the tangential points of $250 \mathrm{~km}$ with a black-red color scale. The 630-nm emission observed by the IMAPNISI is plotted in units of Rayleigh/nm with a blue-white color scale. In $\mathbf{b}$, data of A-IMAP and IMAPNISI along the green line

in $\mathbf{a}$ are presented as functions of latitude. The red solid line is for the total counts of the red channe/ with the summation range from 180 to $300 \mathrm{~km}$ and the red dash line is for that with the summation range from 230 to $300 \mathrm{~km}$. The blue line is for the 630-nm emission observed by the IMAPNISI track was $21 \mathrm{~km}$, which is determined by the $2.75 \mathrm{~s} \mathrm{imag-}$ ing interval of the photographs. The horizontal resolution perpendicular to the track was $19 \mathrm{~km}$, corresponding to the size of 10 pixels of the image. The robot arms of the ISS obstructed the airglow imaging on the right side of the FOV as can be seen in Fig. 1. They cause the two line-shape data gaps on the south side of the mapping image along the ISS orbit. The solar panels of the ISS also obstructed the observation on the left side of the FOV until 13:49:36 UT. The data gap caused by the solar panels can be seen on the north side of the mapping image. An enhancement of the total count of the red channel caused by an enhancement of the 630-nm emission can be seen around the latitude of $5^{\circ} \mathrm{S}$. This enhancement was interpreted to be caused by the EIA, because the magnetic latitude of the enhancement is consistent with the typical magnetic latitude of the EIA (e.g., Watthanasangmechai et al. 2014, 2015). The total counts of the enhancement on the east side of the FOV were larger than those on the west side. This is because of the alignment of the LOS with the EIA, which has a zonally extended structure. Considering the center LOS of the camera pointed southeast with an AOV of 92 at the low-latitude region, the LOS on the east side of the FOV pointed east, and then an integration along the LOS yielded a large count from the EIA. The LOS on the west side of the FOV pointed south and resulted in a less count.

IMAP/VISI is a Visible and near-Infrared Spectral Imager installed on the Exposure Facility of the Japanese Experiment Module on the ISS (Sakanoi et al. 2011). It had a simultaneous observation on August 26, 2014, with the A-IMAP observation. IMAP/VISI observed several emission lines of the airglow, including the $630-\mathrm{nm}$ OI emission with two slit-line FOVs pointing $45^{\circ}$ forward and $45^{\circ}$ backward to nadir. From 13:40:42 to 14:08:57 UT, it observed the 630-nm OI emission with backward FOV and an exposure time of $4.0 \mathrm{~s}$ in "the spectral mode." In the spectral mode, it recorded the spectral shape in the wavelength range around $630 \pm 6 \mathrm{~nm}$ with a resolution of $1.02 \mathrm{pixel} / \mathrm{nm}$. We assumed that the maximum count is in the wavelength range containing both the 630$\mathrm{nm}$ OI emission and background emission and that the minimum count is in the range containing only the background emission. By subtracting the minimum count from the maximum count, the $630-\mathrm{nm}$ airglow emission peak could be retrieved from the observed spectral shape. The $630-\mathrm{nm}$ OI emission in units of Rayleigh/nm was mapped to an altitude of $250 \mathrm{~km}$. The results are presented in Fig. 6a with a blue-white color scale. The width of the FOV across the track was $350 \mathrm{~km}$ at $250 \mathrm{~km}$ in altitude, and the resolutions across and along the track were 14 and $33 \mathrm{~km}$, respectively. An enhancement of the 630-nm emission caused by the EIA enhancement was 
observed by IMAP/VISI in the latitude range from $0^{\circ} \mathrm{S}$ to $10^{\circ} \mathrm{S}$.

To have more detailed comparison of the data, we plotted the data as functions of latitude in Fig. 6b. The total counts of the A-IMAP red channel and the IMAP/ VISI data along the green line shown in Fig. $6 \mathrm{a}$ are plotted with the red and blue lines, respectively. To examine the effect of the vertical summation range, we show the latitudinal profile of the A-IMAP data with the summation range from 230 to $300 \mathrm{~km}$ with the red dash line in addition to that with the summation range from 180 to $300 \mathrm{~km}$. The center of the EIA was located around $5.5^{\circ}$ $\mathrm{S}$ for both observations. Although the enhancement peak locations showed a good coincidence, the width of the enhancement observed by A-IMAP was wider than that observed by IMAP/VISI. We computed a nonlinear least-squares fit for each latitudinal profile to a function $f(x)=A_{0} e^{\frac{z}{2}}+A_{3}+A_{4}$ where $z=\frac{x-A_{1}}{A_{2}}$. Assuming the width of EIA is twice large as the full-width half maxima, we determined the edge of the EIA for each profile. The southern edge for each profile is indicated with the vertical arrow of each line type in Fig. 6 . The southern edge of the EIA in the red channel with the summation range from 180 to $300 \mathrm{~km}$ was wider by about $3.3^{\circ}$ in latitude or $450 \mathrm{~km}$ in distance than that in the IMAP/ VISI observation, while the edge in the red channel with the summation range from 230 to $300 \mathrm{~km}$ was wider by about $2.2^{\circ}$ in latitude or $300 \mathrm{~km}$ in distance that in the IMAP/VISI observation. The wider enhancement structure in the limb count profile of the photographs would be attributed to the horizontally integrated observation path. There was about $520-\mathrm{km}$ horizontal distance between the tangential points in the altitude range from 180 and $300 \mathrm{~km}$ in each image. For the summation range from 230 to $300 \mathrm{~km}$, the horizontal distance between the tangential points is about $330 \mathrm{~km}$. It means that the limb observation integrated the structure horizontally for about 520 or $330 \mathrm{~km}$ depending on its summation range. As a result, the horizontally extended shape of EIA was observed in the A-IMAP data. The difference in the EIA width derived from different summation altitude ranges can be explained by the difference in horizontal distance between the tangential points in each summation range. On the other hand, IMAP/VISI has a relatively vertical integration path and can observe the true horizontal edge of the EIA. Therefore, it is reasonable that the results from different observation geometries have difference in the EIA edge. Although there is a difference in the width of the structure caused by the observation geometry, the EIA enhancement locations from the two observations have a good agreement. This agreement supports the validity of the proposed calibration method.

\section{Conclusions}

A new calibration technique for space-borne airglow photography was developed and confirmed using the other optical measurements. Matching the apparent city light positions on a photograph with the actual city light positions derived from the DMSP-OLS stable night light map in the pinhole camera model, the imaging parameters can be determined. We applied the method to the photographs taken by astronauts on the ISS on August 28,2014 , and evaluated the precision and stability of the calibration. The precision of the derived time lag for the camera clock was $0.3 \mathrm{~s}$, and that of the camera orientation was $0.08^{\circ}$. The precision of the FOV was $0.12^{\circ}$ for the pixel with a distance of 1500 pixels from the center of the image. The calibration result is precise enough for airglow and aurora studies. This calibration technique makes it possible to utilize photographs taken on LEO satellites as a reference for the airglow and aurora structures.

The raw count data of the photographs were mapped to their tangential points in geographical coordinates using the calibrated imaging parameters. The peak heights for the $F$-region airglow, e.g., the 630 and 557.7-nm OI emissions, and the mesospheric airglow, such as $\mathrm{OI}, \mathrm{NaD}$, and $\mathrm{OH}$ emissions, had a good agreement with those of previous studies. The EIA structures of the 630-nm OI emission obtained from the red channel of the photographs and IMAP/VISI data also had good agreement. These agreements support the validity of the calibration method.

\begin{abstract}
Abbreviations
AOV: angle of view; DMSP-OLS: the visible and infrared sensors on the Defense Meteorological Satellite Program satellite; DSLR: digital single-lens reflex; EIA: equatorial ionization anomaly; EXIF format: exchangeable image file format; FOV: field of view; ISS: International Space Station; ISS-IMAP: International Space Station-Ionosphere, Mesosphere upper Atmosphere, and Plasmasphere mapping mission; LEO: low Earth orbit; LOS: line of sight; VISI: Visible and nearInfrared Spectral Imager
\end{abstract}

\section{Authors' contributions}

YH developed the calibration method, applied the method to data, interpreted the calibration result, and wrote the first draft of the manuscript. AS conceived the airglow observation with photographs taken on the International Space Station, coordinated observations, contributed to the improvement of the calibration method. ME lead the sensitivity calibration of the camera. All authors revised and improved the manuscript. All authors read and approved the final manuscript.

\section{Author details}

${ }^{1}$ Graduate School of Science, Kyoto University, Kyoto, Japan. ${ }^{2}$ Department of Polar Science, Graduate University for Advanced Studies (SOKENDAI), Tachikawa, Japan. ${ }^{3}$ National Institute of Polar Research, Tachikawa, Japan.

\section{Acknowledgements}

Data that support this report are from Astronaut-lonosphere, Mesosphere, upper Atmosphere, and Plasmasphere mapping (A-IMAP) mission and the lonosphere, Mesosphere, upper Atmosphere, and Plasmasphere mapping mission from the ISS (ISS-IMAP mission). We thank all the member of the A-IMAP and ISS-IMAP mission. We are also thankful to NOAA's National Geophysical Data Center and US Air Force Weather Agency for the provision of the global nighttime stable light data obtained from the visible and infrared sensors 
(OLS) on the Defense Meteorological Satellite Program (DMSP) satellite. This work was supported by JSPS KAKENHI Grant Number JP26610156.

\section{Competing interests}

The authors declare that they have no competing interests.

Received: 16 June 2016 Accepted: 31 August 2016

Published online: 15 September 2016

\section{References}

Adachi T, Yamaoka M, Yamamoto M et al (2010) Midnight latitude-altitude distribution of $630 \mathrm{~nm}$ airglow in the Asian sector measured with FORMOSAT-2/ISUAL. J Geophys Res 115:1-11. doi:10.1029/2009JA015147

Baker DJ, Stair AT (1988) Rocket measurements of the altitude distributions of the hydroxyl airglow. Phys Scr 37:611-622. doi:10.1088/0031-8949/37/4/021

Bayer B (1976) Color imaging array. US Patent 3971065, 20 July 1976

Broadfoot AL, Bellaire PJ (1999) Bridging the gap between ground-based and space-based observations of the night airglow. J Geophys Res 104:17127. doi:10.1029/1999JA900135

Broadfoot AL, Kendall KR (1968) The airglow spectrum, 3100-10,000 A. J Geophys Res 73:426. doi:10.1029/JA073i001p0042

Brown DC (1971) Close-range camera calibration. Photogramm Eng $37: 855-866$

Chamberlain JW (1958) The blue airglow spectrum. Astrophys J 128:713. doi:10.1086/146582

Jaehne B (1997) Practical handbook on image processing for scientific applications. CRC Press Inc, Boca Raton

Khomich VY, Semenov Al, Shefov NN (2008) Airglow as an indicator of upper atmospheric structure and dynamics. Springer, Berlin

Krassovsky VI, Shefov NN, Yarin VI (1962) Atlas of the airglow spectrum 3000 $12400 \AA$ A. Planet Space Sci 9:883-915. doi:10.1016/0032-0633(62)90008-9
Kubota M, Shiokawa K, Ejiri MK et al (2000) Traveling ionospheric disturbances observed in the Ol 630-nm nightglow images over Japan by using a Multipoint Imager Network during the FRONT Campaign. Geophys Res Lett 27:4037-4040. doi:10.1029/2000GL011858

Liu G, Shepherd GG (2006) An empirical model for the altitude of the $\mathrm{OH}$ nightglow emission. Geophys Res Lett 33:L09805. doi:10.1029/200 5GL025297

López-González MJ, López-Moreno JJ, Rodrigo R (1992) Altitude and vibrational distribution of the $\mathrm{O} 2$ ultraviolet nightglow emissions. Planet Space Sci 40:913-928. doi:10.1016/0032-0633(92)90132-8

Packer DM (1961) Altitude of the night airglow radiations. Ann Geophys 17:67-75

Sakanoi T, Akiya Y, Yamazaki A et al (2011) Imaging observation of the earth's mesosphere, thermosphere and ionosphere by VISI of ISS-IMAP on the International Space Station. IEEJ Trans Fundam Mater 131:983-988. doi:10.1541/ieejfms.131.983

Takahashi H, Clemesha BR, Batista PP et al (1990) Equatorial f-region oi 6300 å and oi 5577 å emission profiles observed by rocketborne airglow photometers. Planet Space Sci 38:547-554. doi:10.1016/0032-0633(90)90148-J

The Gateway to Astronaut Photography of Earth (1995). http://eol.jsc.nasa. gov/. Accessed 6 Apr 2016

Trucco E, Verri A (1998) Introductory techniques for 3-D computer vision. Prentice Hall, Englewood Cliffs

Version 4 DMSP-OLS Nighttime Lights Time Series (2015). http://ngdc.noaa. gov/eog/dmsp/downloadV4composites.html. Accessed 6 Apr 2016

Watthanasangmechai K, Yamamoto M, Saito A et al (2014) Latitudinal GRBRTEC estimation in Southeast Asia region based on the two-station method. Radio Sci 49:910-920. doi:10.1002/2013RS005347

Watthanasangmechai K, Yamamoto M, Saito A et al (2015) Temporal change of EIA asymmetry revealed by a beacon receiver network in Southeast Asia. Earth Planets Space 67:75. doi:10.1186/s40623-015-0252-9

\section{Submit your manuscript to a SpringerOpen ${ }^{\odot}$ journal and benefit from:}

- Convenient online submission

- Rigorous peer review

- Immediate publication on acceptance

- Open access: articles freely available online

- High visibility within the field

- Retaining the copyright to your article

Submit your next manuscript at springeropen.com 